\section{Preliminary monitoring of the presence of perfluoroalkyl substances in Italian eggs from different breeding systems}

\author{
Elisa Ghelli, ${ }^{1}$ Maria Teresa Tondo, ${ }^{1}$ \\ Elisa Zironi, ${ }^{1}$ Giampiero Pagliuca, ${ }^{1}$ \\ Federico Sirri, ${ }^{2}$ Teresa Gazzotti ${ }^{1}$
}

${ }^{1}$ Department of Veterinary Medical Sciences and ${ }^{2}$ Department of Food Science and Technology, University of Bologna, Ozzano dell'Emilia, Italy

\begin{abstract}
Perfluoroalkylated substances (PFASs) are a wide cluster of fluorinated molecules largely engaged industrially and commercially for many purposes. Because of the strength of the fluorine-carbon bond, PFASs show a firm tenacity against thermal degradation, hydrolysis, photolysis and biodegradation. On the other hand, such chemical stability gives them persistent environmental pollutant feature. In 2012, EFSA published a scientific report on PFASs in food, mentioning their adverse effects on health. Based on observational studies evidences, EFSA has recommended a tolerable daily intake (TDI) for the two most known PFASs, i.e. PFOS $150 \mathrm{ng} / \mathrm{kg}$ b.w./day and PFOA $1500 \mathrm{ng} / \mathrm{kg}$ b.w./day. The aim of this study was to monitor, for the first time, the level of contamination of PFASs in chicken eggs laid in Northern Italy. The eggs were collected from different rearing systems, in order to search a correlation between this variable and the contamination of PFASs. In this study four PFASs [perfluoro-nnonanoic acid (PFNA), perfluoro-noctanoic-acid (PFOA), sodium perfluoro-1hexanesulfonate (PFHxS) and sodium perfluoro-1-octanesulfonate (PFOS)] were analyzed by liquid chromatography-tandem mass spectrometer (LC-MS/MS). 132 eggs were analyzed, split up in 11 groups according to the geographical origin and rearing system. Results accord with literature data available for chicken eggs: almost all the samples show a PFASs contamination level under the limit of quantification (LOQ) of $0.25 \mathrm{ng} / \mathrm{mL}$. No significant difference results from the rearing system, attesting an equal distribution and a concentration of PFASs detectable under the limit of quantification.
\end{abstract}

Correspondence: Elisa Ghelli, Laboratory of Analytical Bio-Agroalimentary Chemistry (CABA-Lab), Department of Veterinary Sciences, Alma Mater Studiorum - University of Bologna, via Tolara di Sopra 50, 40064 Ozzano dell'Emilia (BO), Italy.

Tel. +39.340.4092343.

E-mail: elisa.ghelli2@unibo.it

Key words: PFASs, chicken eggs, UPLCMS/MS

Contributions: the authors contributed equally.

Conflict of interests: the authors declare no potential conflict of interests.

Funding: none.

Received for publication: 16 July 2018.

Revision received: 20 March 2019.

Accepted for publication: 12 April 2019

This work is licensed under a Creative Commons Attribution-NonCommercial 4.0 International License (CC BY-NC 4.0).

(C) Copyright E. Ghelli et al., 2019

Licensee PAGEPress, Italy

Italian Journal of Food Safety 2019; 8:7702

doi:10.4081/ijfs.2019.7702 cides (3M Company, 1999; Lindstrom et al., 2011). In 2012, EFSA, the European Food Safety Authority, published a scientific report on PFAS in food, mentioning their adverse effects on health based on several studies on experimental animals, i.e. hepatotoxicity, developmental and reproductive toxicity, neurobehavioral toxicity, immunotoxicity, lung toxicity and endocrine disruptors (EFSA, 2012). For this reason, EFSA has recommended a tolerable daily intake (TDI) for the two most known PFAS, i.e. PFOS $150 \mathrm{ng} / \mathrm{kg}$ b.w./day and PFOA 1500 $\mathrm{ng} / \mathrm{kg}$ b.w./day, and additionally recommended to the scientific community to collect more data on PFAS levels in food (EFSA, 2012).

Therefore, except for PFAS industrial workers, diet is considered the main exposure route to PFAS (Jain, 2018). For this reason, many scientific papers have been published with the purpose to evaluate the PFAS level contamination in different food commodities. From literature it emerged that fish and seafood in general are the most contaminated food categories (EFSA, 2012; Vestergren et al., 2012; Hlouskova et al., 2013; Barbarossa et al., 2016; Jian et al., 2017). In a recent study, it was evidenced that there is a PFAS level affinity within foodstuffs that is showed in the following order: fish and shellfish > eggs and meat products $>$ milk products and beverages $>$ vegetables (Jian et al., 2017). This trend is explainable by their protein affinity, the high bioaccumulation potential and bio- magnification effect of these resistant pollutant in the food chain (Jian et al., 2017).

Chicken eggs play an important role in human diet, due to their content of high biological value proteins combined with low cost and lack of religious and ethical restrictions. In Italy their consumption is relevant, and is about 215 eggs per person per year (Unaitalia, 2017) corresponding to about $13.5 \mathrm{~kg}$ per person per year. A part of the consumption of these eggs occurs indirectly with the intake of foods, such as egg pasta, sweets, biscuits and bakery products. Despite this scenario, information on their contamination by PFAS is still very low. Only few scientific studies have been carried out on the contamination of chicken eggs (D'Hollander et al., 2011; Zafeiraki et al., 2016), while most of the scientific works concern the monitoring of PFAS in wild bird eggs (Miller et al., 2015; Letcher et al., 2015) or other wild animal species related to environmental biomonitoring studies. Otherwise some studies report PFAS levels in different food items amongst which also egg, but in a limited number, considering a wide foodstuff range (Guerranti et al., 2013; Hlouskova et al., 2013; Jain, 2018; Jian et al., 2017; Vestergren et al., 2012). In Italy, a few scientific works on the monitoring of PFAS have been conducted on food matrices different from chicken eggs (Farabegoli et al., 
2013; Barbarossa et al., 2014), so there are currently no studies on PFAS monitoring in chicken eggs.

The aim of this study was to monitor, for the first time, the contamination of PFASs in commercial Italian chicken eggs, obtained from different hen rearing system. In this study the four PFAS more frequently studied were considered: perfluoro-nnonanoic acid (PFNA), perfluoro-noctanoic-acid (PFOA), sodium perfluoro-1hexanesulfonate (PFHxS) and sodium perfluoro-1-octanesulfonate (PFOS) by ultraperformance liquid chromatography coupled to tandem mass spectrometry (UPLCMS/MS).

\section{Materials and Methods}

\section{Sample collection}

The egg samples were collected from commercial laying hen farms in 2017. Sampling was based on the following variables: rearing system (organic, aviary system, battery cage and barn) and geographical origin of the eggs. A total of 132 eggs were collected, divided into 11 groups based on the variables listed in Table 1 . After sampling, eggs were boiled and the yolks were separated from egg white. Four pools (containing three homogenized yolks) were created for each group, for a total of 44 samples analyzed. The pools were stored at $-20^{\circ} \mathrm{C}$ until the analysis.

\section{Reagents and chemicals}

The PFNA, PFOA, PFHxS and PFOS standards and their respective labeled standards (IS), perfluoro-n- $\left(1,2,3,4,5-{ }^{13} \mathrm{C}_{5}\right)$ nonanoic acid (M-PFNA), perfluoro-n$\left(1,2,3,4-{ }^{13} \mathrm{C}_{4}\right)$ octanoic acid (M-PFOA), perfluoro-1-hexane $\left({ }^{18} \mathrm{O}_{2}\right)$ sulfonate (MPFHxS), sodium perfluoro-1-(1,2,3,4- $\left.{ }^{13} \mathrm{C}_{4}\right)$ octanesulfonate (M-PFOS) were purchased from Wellington (Guelph, Ontario, Canada). An appropriate amount of each labeled standard was combined and diluted with methanol to obtain a "IS working solution" at a concentration of $50 \mathrm{ng} / \mathrm{mL}$. The same procedure was used to prepare a "PFASs working solution".

Methanol, ammonium acetate, acetonitrile and formic acid were all of mass spectrometry grade and were all from Fluka (Honeywell). Sodium hydroxide pellets (>98\%) were from Fluka (Honeywell), sodium acetate and ammonium hydroxide (33\%) were both from Sigma-Aldrich (St. Louis, MO, USA). Hydrochloric acid (37\%) was from Carlo Erba Reagents (Cornaredo, MI, Italy). The solid-phase extraction (SPE) cartridges were Oasis WAX $3 \mathrm{cc}, 60 \mathrm{mg}$
(Waters Corp., Milford, MA, USA). Ultrapure water $(18.2 \mathrm{M} \Omega / \mathrm{cm})$ was obtained via a Human Power I lab water purification system (Human Corp., Seoul, South Korea).

\section{Sample preparation}

Samples were extracted adopting to the method of Zafeiraki (Zafeiraki et al., 2016). The procedure was carried using polypropylene (PP) materials, to avoid the interaction with glass.

Briefly, $50 \mu \mathrm{L}$ of "IS working solution" was added at $1 \mathrm{~g}$ of homogenized yolk for quantification. Later, the sample was digested by an alkaline solution $(2 \mathrm{~mL}$ of sodium hydroxide $200 \mathrm{mM}$ ) and homogenized by Ultra Turrax for 1 minute. After that, $10 \mathrm{~mL}$ of methanol were added for extraction and then agitated by magnetic stirrer for $30 \mathrm{~min}$ utes. Afterwards $150 \mu \mathrm{L}$ of $\mathrm{HCl}(37 \%)$ were added and the sample centrifugated for 10 minutes at $10.000 \mathrm{rpm}$, then the supernatant was transferred into a tube containing 25 $\mathrm{mL}$ of ultrapure water. The extract was purified by SPE Oasis WAX (Weak Anionic eXchange) cartridges. The cartridge was conditioned with $4 \mathrm{~mL}$ of methanol and 4 $\mathrm{mL}$ of water before sample loading. After a wash by $4 \mathrm{~mL}$ of $25 \mathrm{mM}$ sodium acetate buffer (adjusted to $\mathrm{pH} 4$ with hydrochloric acid), the elution was obtained by $2 \mathrm{~mL}$ of $2 \%$ ammonium hydroxide solution in acetonitrile. Finally, the eluate was dried by gentle $\mathrm{N}_{2}$ flow at $45^{\circ} \mathrm{C}$ and redissolved with $300 \mu \mathrm{L}$ of $20 \mathrm{mM}$ ammonium acetate : methanol $(90: 10)$ and then transferred into a vial for UPLC-MS/MS analysis.

A calibration curve was prepared with 1 $\mathrm{g}$ of blank yolk which was spiked with appropriate amounts of the "PFASs working solution" to obtain 5 levels of concentration $(0,0.5,1,5,10 \mathrm{ng} / \mathrm{g})$ and $50 \mu \mathrm{L}$ of the internal standard IS PFASs working solution $(50 \mathrm{ng} / \mathrm{mL})$. In addition, quality control (QC) samples at three concentrations $(0.5,2$, and $5 \mathrm{ng} / \mathrm{g})$ were used to mon- itor the performance of the method.

Limits of quantification (LOQs) and limits of detection (LODs) of the method, defined as the concentrations providing a chromatographic signal with a signal-tonoise $(\mathrm{S} / \mathrm{N})$ ratio equal to 10 and 3 respectively, were $0.25 \mathrm{ng} / \mathrm{g}$ and $0.1 \mathrm{ng} / \mathrm{g}$ for all analytes.

\section{Analytical conditions}

The separation was achieved by an Acquity ultra-performance liquid chromatographic system consisting of a binary pump, solvent degasser, autosampler and column heater fitted with a Waters BEH C18 column $(1.7 \mu \mathrm{m}, 2.1 \times 50 \mathrm{~mm})$ equipped with a guard column (Waters Corporation, Milford, MA, USA). In order to avoid contamination by LC system an isolator column for PFAS $(2.1 \times 50 \mathrm{~mm})$ (Waters Corporation, Milford, MA, USA) was used for all the analysis. The mobile phase consisted of $20 \mathrm{mM}$ ammonium acetate aqueous solution (A) and methanol (B); the chromatographic gradient was operated at a flow rate of $0.4 \mathrm{~mL} / \mathrm{min}$ starting from 0 min: $90 \%$ A, 10\% B; 1,50 min: $90 \%$ A, 10\% B; $2,50 \min 20 \% \mathrm{~A}, 80 \% \mathrm{~B}, 4 \min 20 \% \mathrm{~A}$, $80 \% \mathrm{~B} ; 4,50 \mathrm{~min}: 90 \% \mathrm{~A}, 10 \% \mathrm{~B} ; 6 \mathrm{~min}$ : $90 \% \mathrm{~A}, 10 \% \mathrm{~B}$. The mass spectrometer was a Quattro Premiere XE, a triple quadrupole instrument equipped with an ESCITM MultiMode Ionization Source (Waters Corporation).

The whole analysis was performed in ESI- mode using multiple reaction monitoring (MRM). The monitored transitions for each analyte were reported in Table 2.

The capillary voltage was $2.0 \mathrm{kV}$, extractor voltage $2.00 \mathrm{~V}$, source temperature $150^{\circ} \mathrm{C}$, and desolvation temperature $450^{\circ} \mathrm{C}$. Nitrogen was used as desolvation gas 700 $\mathrm{L} / \mathrm{hr}$ and cone gas $100 \mathrm{~L} / \mathrm{hr}$, whereas the collision gas was argon. Data acquisition processing was performed using Mass Lynx 4.1 software (Waters Corp.).

Table 1. Eggs sampling based on different rearing systems and different geographical origin.

\begin{tabular}{lccc} 
Group & $\mathbb{N}^{\circ}$ pool & Rearing system & Geographical origin \\
A & 4 & Barn & Pavia \\
B & 4 & Organic & Verona \\
\hline C & 4 & Battery cage & Forlì-Cesena \\
D & 4 & Barn & Bologna \\
\hline E & 4 & Battery cage & Forlì-Cesena \\
F & 4 & Aviary system & Ravenna \\
\hline G & 4 & Aviary system & Ravenna \\
H & 4 & Organic & Bologna \\
\hline I & 4 & Battery cage & Romagna \\
L & 4 & Organic & Romagna \\
\hline M & 4 & Barn & Romagna
\end{tabular}




\section{Results and Discussion}

PFAS were determined in the yolk, because in literature earlier works reported that PFAS are primarily found in the egg yolk than in the egg white (Zafeiraki et al., 2016).

In all samples the level of each analyte was under the LOQ of $0.25 \mathrm{ng} / \mathrm{mL}$, except for two samples belonging to group A, obtained from barn, that showed contamination of PFOS in one sample and of PFHxS in the other, both at the level of $0.4 \mathrm{ng} / \mathrm{g}$. Levels of PFOA, PFNA and PFHxS, between the LOD $(0.1 \mathrm{ng} / \mathrm{g})$ and LOQ, were found in the $15.9 \%$ of sample (Table 3 ). PFOS was detected only in one sample, but at quantifiable level, while in many works PFOS is the most frequently found PFAS and the most abundant (Zafeiraki et al., 2016).

This very low uniform distribution of PFASs in commercial eggs is in accordance with the few data reported in literature, in particular with Zafeiraki's work on Greek and Netherlands commercial eggs. Furthermore, the low contamination of commercial eggs is in contrast to the high levels found in home produced eggs as reported in literature (D'Hollander et al., 2011; Zafeiraki et al., 2016).

The origin of the sampled eggs depending on the rearing system was the following: $27 \%$ organic, $27 \%$ battery cage, $27 \%$ barn and $19 \%$ aviary system. Regarding the rearing system of hen eggs production, the levels of contamination were so limited, that no difference emerged.

As far as the geographical origin is concerned, the irregular sampling does not allow to draw a correct correlation between this variable and results.

\section{Conclusions}

In this preliminary study, commercially Italian chicken eggs were investigated for the first time in order to monitor their level of PFASs contamination analyzing the four most widespread substances (PFOS, PFOA, PFNA, PFHxS). Data show a very low and uniform contamination in commercial eggs regardless the rearing system and origin. Only two samples show a quantifiable contamination of PFOS and of PFHxS.

Considering the very low level of PFAS contamination measured in the present study and the TDI established, it could be concluded that the consumption of Italian commercial eggs does not pose a high risk for consumers.
Table 2. Mass spectrometry parameters for the selected PFASs.

\begin{tabular}{lcccc} 
Compound & $\begin{array}{c}\text { Precursorion } \\
(\mathrm{m} / \mathbf{z})\end{array}$ & $\begin{array}{c}\text { Productions } \\
(\mathrm{m} / \mathbf{z})\end{array}$ & $\begin{array}{c}\text { Cone Voltage } \\
(\mathrm{kV})\end{array}$ & $\begin{array}{c}\text { Collision Energy } \\
(\mathrm{eV})\end{array}$ \\
PFOS & 498.50 & 99.10 & 50 & 35 \\
& & 80.20 & 50 & 40 \\
PFHxS & 398.60 & 99.10 & 52 & 35 \\
& & 80.20 & 52 & 38 \\
\hline PFNA & 462.50 & 419.00 & 12 & 10 \\
& & 219.10 & 12 & 16 \\
PFOA & 412.60 & 368.90 & 12 & 9 \\
& & 169.00 & 12 & 17 \\
\hline M-PFOS & 502.50 & 99.10 & 50 & 35 \\
& & 80.20 & 50 & 32 \\
M-PFHXS & 402.60 & 103.10 & 55 & 35 \\
& & 84.20 & 55 & 10 \\
M-PFNA & 467.50 & 423.00 & 12 & 16 \\
& & 219.10 & 12 & 10 \\
M-PFOA & 416.50 & 372.00 & 12 & 17 \\
& & 169.00 & 12 & \\
\hline
\end{tabular}

Table 3. Results of PFASs contamination level in analyzed hen eggs. Traces: value between limit of detection (LOD) and limit of quantification (LOQ).

\begin{tabular}{|c|c|c|c|c|c|}
\hline Group & Pool & PFOA & PFHxS & PFOS & PFNA \\
\hline \multirow[t]{4}{*}{ A } & 1 & traces & - & $0.4 \mathrm{ng} / \mathrm{g}$ & - \\
\hline & 2 & traces & traces & - & - \\
\hline & 3 & - & $0.4 \mathrm{ng} / \mathrm{g}$ & - & - \\
\hline & 4 & - & - & - & - \\
\hline \multirow[t]{4}{*}{ B } & 1 & - & - & - & - \\
\hline & 2 & traces & - & - & - \\
\hline & 3 & - & - & - & - \\
\hline & 4 & - & traces & - & - \\
\hline \multirow[t]{4}{*}{$\mathrm{C}$} & 1 & - & - & - & - \\
\hline & 2 & - & - & - & traces \\
\hline & 3 & - & - & - & - \\
\hline & 4 & - & - & - & - \\
\hline \multirow[t]{4}{*}{ D } & 1 & - & - & - & - \\
\hline & 2 & - & - & - & - \\
\hline & 3 & - & - & - & - \\
\hline & 4 & - & - & - & traces \\
\hline \multirow[t]{4}{*}{$\mathrm{E}$} & 1 & - & - & - & - \\
\hline & 2 & - & - & - & - \\
\hline & 3 & - & - & - & - \\
\hline & 4 & - & - & - & - \\
\hline \multirow[t]{4}{*}{$\mathrm{F}$} & 1 & - & - & - & - \\
\hline & 2 & - & - & - & - \\
\hline & 3 & - & - & - & - \\
\hline & 4 & - & - & - & - \\
\hline \multirow[t]{4}{*}{$\mathrm{G}$} & 1 & - & - & - & - \\
\hline & 2 & - & - & - & - \\
\hline & 3 & - & - & - & - \\
\hline & 4 & - & - & - & - \\
\hline \multirow[t]{4}{*}{$\mathrm{H}$} & 1 & - & - & - & - \\
\hline & 2 & - & - & - & - \\
\hline & 3 & - & - & - & - \\
\hline & 4 & - & - & - & - \\
\hline \multirow[t]{4}{*}{ I } & 1 & - & - & - & - \\
\hline & 2 & traces & traces & - & - \\
\hline & 3 & - & - & - & - \\
\hline & 4 & - & - & - & - \\
\hline \multirow[t]{4}{*}{$\mathrm{L}$} & 1 & - & - & - & - \\
\hline & 2 & - & - & - & - \\
\hline & 3 & - & - & - & - \\
\hline & 4 & - & - & - & - \\
\hline \multirow[t]{4}{*}{ M } & 1 & - & - & - & - \\
\hline & 2 & - & - & - & - \\
\hline & 3 & - & - & - & - \\
\hline & 4 & - & - & - & - \\
\hline
\end{tabular}




\section{References}

3M Company, 1999. Fluorochemical use, distribution and release overview. Available from: http://www.chemicalindustryarchives.org/

Barbarossa A, Gazzotti T, Zironi E, Serraino A, Pagliuca G, 2014. Short communication: Monitoring the presence of perfluoroalkyl substances in Italian cow milk. J Dairy Sci 97:333943.

Bräunig J, Baduel C, Heffernan A, Rotander A, Donaldson E, Mueller JF, 2017. Fate and redistribution of perfluoroalkyl acids through AFFF-impacted groundwater. Sci Total Environ 596-597:3608.

Buck RC, Franklin J, Berger U, Conder JM, Cousins IT, de Voogt P, Jensen AA, Kannan K, Mabury SA, van Leeuwen SP, 2011. Perfluoroalkyl and polyfluoroalkyl substances in the environment: Terminology, classification, and origins. Integr Environ Assess Manag 7:513-41.

D'Hollander W, de Voogt P, Bervoets L, 2011. Accumulation of perfluorinated chemicals in Belgian home-produced chicken eggs. Organohalogen Compd 73:917-20.

EFSA, 2012. Perfluoroalkylated substances in food: occurrence and dietary exposure. EFSA J. 10 Available from: http://doi.wiley.com/10.2903/j.efsa.201 2.2743

Farabegoli F, Barbarossa A, Devicienti C,
Scardilli M, Zironi E, Pirini M, Badiani A, Pagliuca G, Gazzotti T, 2013. Preliminary investigation by liquid chromatography tandem mass spectrometry of perfluorinated compounds presence in bass reared and fished in Italy. Ital J Food Saf 2:e48.

Guerranti C, Perra G, Corsolini S, Focardi SE, 2013. Pilot study on levels of perfluorooctane sulfonic acid (PFOS) and perfluorooctanoic acid (PFOA) in selected foodstuffs and human milk from Italy. Food Chem 140:197-203.

Hlouskova V, Hradkova P, Poustka J, Brambilla G, De Filipps SP, D'Hollander W, Bervoets L, Herzke D, Huber S, de Voogt P, Pulkrabova J, 2013. Occurrence of perfluoroalkyl substances (PFASs) in various food items of animal origin collected in four European countries. Food Addit Contam Part A 30:1918-32.

Jain RB, 2018. Contribution of diet and other factors to the observed levels of selected perfluoroalkyl acids in serum among US children aged 3-11 years. Environ Res 161:268-75.

Jian J-M, Guo Y, Zeng L, Liang-Ying L, Lu X, Wang F, Zeng EY, 2017. Global distribution of perfluorochemicals (PFCs) in potential human exposure source-a review. Environ Int 108:51-62.

Lau C, Butenhoff JL, Rogers JM, 2004. The developmental toxicity of perfluoroalkyl acids and their derivatives. Toxicol Appl Pharmacol 198:231-41.

Letcher RJ, Su G, Moore JN, Williams LL, Martin PA, de Solla SR, Bowerman
WW, 2015. Perfluorinated sulfonate and carboxylate compounds and precursors in herring gull eggs from across the Laurentian Great Lakes of North America: Temporal and recent spatial comparisons and exposure implications. Sci Total Environ 538:468-77.

Lindstrom AB, Strynar MJ, Libelo EL, 2011. Polyfluorinated Compounds: Past, Present, and Future. Environ Sci Technol 45:7954-61.

Miller A, Elliott JE, Elliott KH, Lee S, Cyr F, 2015. Temporal trends of perfluoroalkyl substances (PFAS) in eggs of coastal and offshore birds: increasing PFAS levels associated with offshore bird species breeding on the Pacific coast of Canada and wintering near Asia. Environ Toxicol Chem 34:1799808.

Unaitalia, 2017. Annata avicola 2017. Available from: http://www.unaitalia.com/mercato/anna ta-avicola/

Vestergren R, Berger U, Glynn A, Cousins IT, 2012. Dietary exposure to perfluoroalkyl acids for the Swedish population in 1999, 2005 and 2010. Environ Int 49:120-7.

Zafeiraki E, Costopoulou D, Vassiliadou I, Leondiadis L, Dassenakis E, Hoogenboom RLAP, van Leeuwen SPJ, 2016. Perfluoroalkylated substances (PFASs) in home and commercially produced chicken eggs from the Netherlands and Greece. Chemosphere 144:2106-12. 